\title{
Profound Hypotension in L2 Burst Fracture Patient Associated with Superior Gluteal Artery Rupture
}

\author{
Jaesung Han, Inbo Han \\ Department of Neurosurgery, CHA Bundang Medical Center, CHA University, Seongnam, Korea
}

\begin{abstract}
Rupture of the superior gluteal artery (SGA) is a rare complication. The SGA is vulnerable to injury during pelvic trauma due to its position in the sciatic notch. We report an unusual case of a successfully embolized SGA rupture in L2 burst fracture patient with blunt buttock contusion without associated pelvic fracture. A 45-year-old male was presented to the emergency room (ER) with L2 burst fracture showing a hypovolemic shock state. A computed tomography (CT) scan of the abdomen and pelvis revealed diffuse swelling of the right gluteus muscles with highly attenuated massive hematoma. Emergency pelvic angiography revealed a rupture of the right SGA. Sequential embolization was performed successfully. The patient underwent decompressive L2 total laminectomy with posterior fusion after stabilization of vital sign. The patient became neurologically improved after the operation. Although SGA injury is rare in L2 burst fracture associated with blunt trauma to the pelvis, the presence of hypotensive shock with clinical symptoms warrants the investigation of these vascular injuries. Selective angiography and embolization is considered the treatment of choice.
\end{abstract}

Key Words: Spinal fracture $\cdot$ Embolization $\cdot$ Angiography

\section{INTRODUCTION}

Superior gluteal artery (SGA) injury is a rare but well known complication of blunt trauma usually associated with pelvic and acetabular fractures ${ }^{2,3)}$. Other causes include penetrating trauma, iatrogenic injury during iliosacral screw fixation or traction injury in high energy trauma ${ }^{2}$. The SGA is vulnerable to injury during pelvic trauma due to its position in the sciatic notch $^{2)}$.

SGA injury typically presents as a painful buttock mass and may be pulsatile and have a systolic bruit. Clinical symptoms may be obscured for weeks to months after the initial injury ${ }^{10)}$. Early detection and embolization of this arterial injury should prevent further complications such as hypovolemic shock and compartment syndrome resulting in surgical interventions. Delay in diagnosis leads to significant morbidity and mortality.

The following case report describes rupture of the SGA with isolated L2 bursting fracture secondary to blunt trauma as the only associated etiology. The objective of this report is to raise awareness of the association between isolated $\mathrm{L} 2$ burst

Corresponding author: Inbo Han

Department of Neurosurgery, Spine Center, CHA Bundang Medical Center,

CHA University, 59, Yatap-ro, Bundang-gu, Seongnam 13496, Korea

Tel: +82-31-780-1924, Fax: +82-31-780-5269

E-mail: hanib@cha.ac.kr fracture and the SGA rupture by describing the clinical features and highlighting importance of rapid diagnosis and treatment thus preventing further complications.

\section{CASE REPORT}

A healthy 45-year-old male fell from a height of $5 \mathrm{~m}$ with direct collision of the buttock to the ground. The patient complained of severe lower back pain with weakness and hypoesthesia of bilateral lower extremities. Upon admission to the emergency department, primary assessment ruled out any immediate life threatening injuries. No signs of external bleeding, hematoma, or skin compromise were observed. However, hypotension and tachycardia with blood pressure $79 / 44 \mathrm{mmHg}$ and pulse rate of 135 beats per min were recorded despite a stable $\mathrm{O}_{2}$ saturation of $97 \%$ without oxygen supply. The initial hemoglobin level was $10.8 \mathrm{~g} / \mathrm{dL}$. Physical examination revealed weakness along the bilateral lower extremities with poor grade from the hip flexors to the ankle dorsi-flexors. Hypoesthesia to light touch and pinprick was observed below the L2 dermatome. Deep tendon reflexes were hypo-reflexive and no pathologic reflexes were observed. A plain radiograph of the pelvis and hip showed no abnormalities but a lumbar spine film revealed isolated L2 body burst fracture causing central canal stenosis. The patient was clinically diagnosed with isolated L2 body burst fracture. The patient was prepared for an emergency operation of decompressive L2 laminectomy 

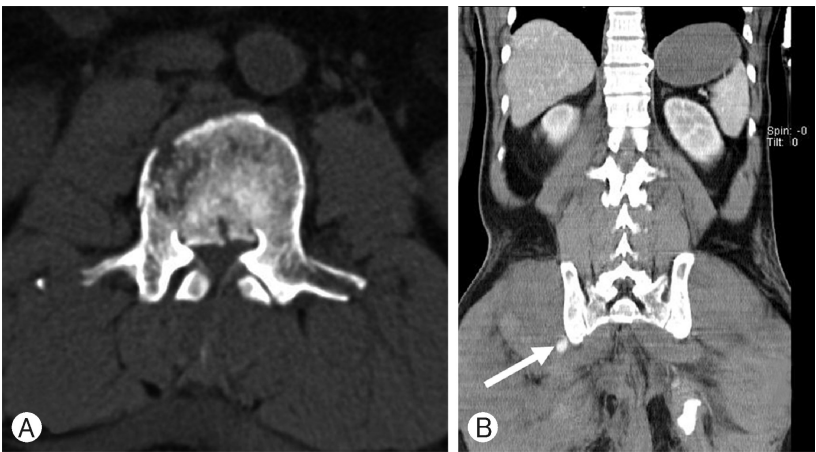

Fig. 1. Abdomen-pelvic computed tomography. Burst fracture of L2 body is noted with narrowing of central spinal canal (A). A small-sized nodular enhancing lesion is noted in the right gluteus muscle in portal phase (white arrow). It is expanded to surrounding muscle in delayed phase (B).
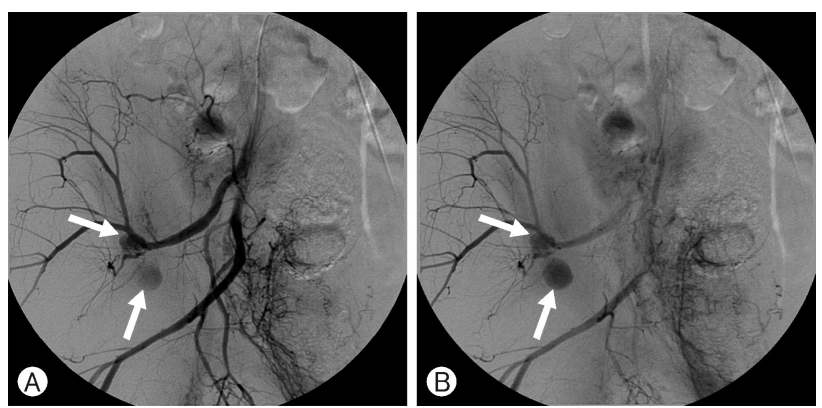

Fig. 2. Internal iliac angiography, pre-embolization. Pre-embolization of right internal iliac arteriography demonstrating active bleeding with pseudoaneurysm (white arrows) of right superior gluteal artery (A, B).

\section{and posterior fusion.}

However, despite aggressive fluid resuscitation during the pre-operative period, persistent hypotension was observed and diagnostic imaging for obscure bleeding was done with a contrast enhanced spine computed tomography (CT) scan. The scan revealed isolated L2 body and right side pedicle fractures with bony protrusions to the posterior side resulting in central canal stenosis along with highly attenuated massive hematoma at the right gluteal muscles with diffuse swelling of the surrounding musculature (Fig. 1). An emergency internal iliac arteriography was done, revealing active bleeding point from the SGA with formation of pseudoaneurysm (Fig. 2). The patient subsequently underwent satisfactory selective embolization using four $4 \times 30 \mathrm{~mm}$ coils and one $5 \times 40 \mathrm{~mm}$ coil (Fig. 3). A repeat hemoglobin measurement after embolization was 7.9 $\mathrm{g} / \mathrm{dL}$, and the patient was transfused with two units of crossmatched, packed red blood cells. His hemodynamic status reco vered to the normal range within the following hr. After successful embolization, decompressive L2 total laminectomy with
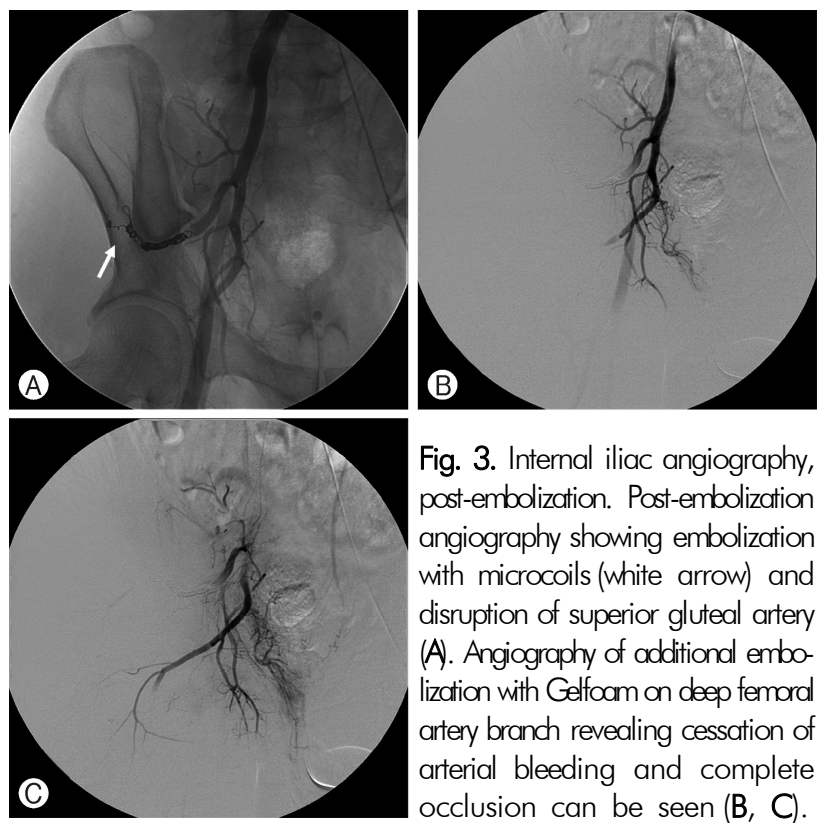

Fig. 3. Internal iliac angiography, post-embolization. Post-embolization angiography showing embolization with microcoils (white arrow) and disruption of superior gluteal artery (A). Angiography of additional embolization with Gelfoam on deep femoral artery branch revealing cessation of arterial bleeding and complete occlusion can be seen ( $B, C)$.
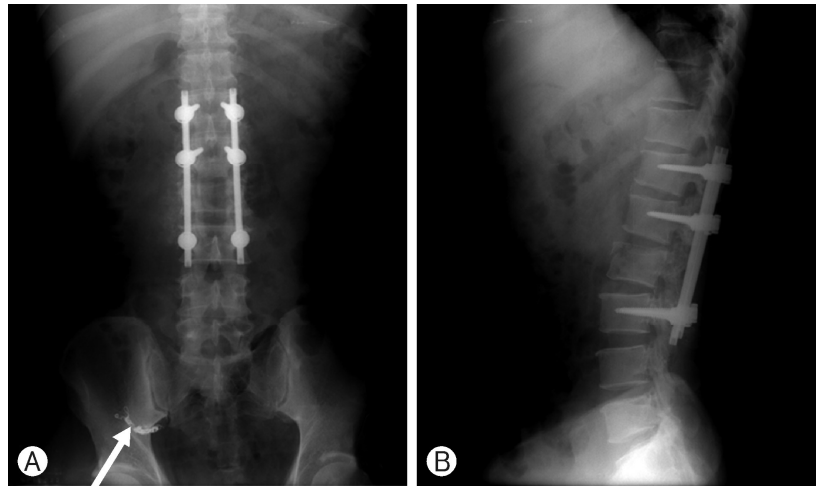

Fig. 4. Postoperative lumbar spine X-ray. Postoperative plain radiographs revealing $\mathrm{L} 2$ total laminectomy and posterior fixation with pedicle screws and rods. Also microcoils are noted in the right superior gluteal artery (white arrow) (A, B).

posterior fusion was performed (Fig. 4). The patient's lower extremity motor grades improved to good grade and hypoesthesia was no longer observed following operation. At 6months follow-up, the patient re-visited our outpatient clinic with an independent outdoor gait.

\section{DISCUSSION}

The SGA has been reported as the most commonly affected vessel associated with posterior pelvic fractures in acute injuries. Sheridan et al. ${ }^{7}$ assessed CT scans of 104 consecutive patients who had sustained a traumatic pelvic fracture and undergone emergent pelvic angiography, finding that $61(58 \%)$ of the 
patients had arterial bleeding at the time of the angiography and that the SGA is one of the most commonly injured arteries.

The SGA is the largest posterior branch of the internal iliac artery and lies between the lumbosacral trunk and first sacral ventral ramus or between the first and second sacral rami. It exits the pelvis through the greater sciatic foramen, passes over the piriformis muscle and splits into superficial and deep branches. As the SGA exits the pelvis through the greater sciatic foramen, it crosses immediately below the sacroiliac joint and this hazardous position makes it vulnerable to disruption, spasm, stretching, or intimal tear in any blunt pelvic trauma that results in disruption of the sacroiliac joint ${ }^{9}$. Rupture of the SGA is frequently associated with pelvic fractures, hip dislocations and penetrating injuries ${ }^{2)}$.

In previous published literatures, most of the SGA injuries occurred as a result of pelvic and acetabular trauma accompanied with pelvic bone fracture ${ }^{2,3)}$. We present a patient with rupture of the SGA with an isolated L2 burst fracture without pelvic bone fracture. To our knowledge, there have been three other case reports of injuries to the SGA following blunt trauma without an associated pelvic fracture. In these cases, the mechanism of the SGA injury was shearing forces at the origin of the SGA in the sciatic notch. This mechanism can also explain the reason why the SGA rupture occurred without pelvic fracture in our case $\mathrm{e}^{2,5)}$.

Unstable hemodynamic status of a patient who has traumatic spine fracture without pelvic bone fracture was thought to be due to possible intra-abdominal cause such as injury of inferior vena cava or aorta rather than a pelvic bleed like the SGA injury. But if spine fracture is caused by buttock contusion like our case, the SGA injury which is vulnerable vessel in pelvic anatomy should be suspected in spite of the absence of pelvic fracture. Especially buttock color change or painful swelling is suggestive of the gluteal compartment syndrome due to the SGA injury ${ }^{2,5)}$.

Extensive bleeding from the SGA may result in compartment syndrome of the buttock leading to sciatic neuropathy, although this tends to occur gradually and presents weeks to months after the initial injury. Untreated compartment syndromes have serious complications resulting in necrosis of the muscles and nerves, systemic acidosis, rhabdomyolysis with myoglobinuria, subsequent renal failure and possibly death. Some reports in the literature suggest ischemia over $4 \mathrm{hr}$ duration can cause irreversible muscle damage ${ }^{8)}$.

The patient in our case benefited from early suspicion of active bleeding and received early diagnostic imaging leading to early intervention for the condition and resulting in early recovery and minimal long-term complications. A CT scan with contrast potentially allows surgeons to distinguish high or low flow hemorrhage and helps detect the location of the hemor- rhage site and involved branches. This is not limited to only the SGA rupture, but also hemorrhage from either inferior vena cava or aorta that more close to lumbar spine ${ }^{7}$. However, angiography is the diagnosis of choice as immediate embolization of the lesion is possible upon confirmation ${ }^{6}$. A routine aortic flush angiogram may be insufficient as it may show normal aortoiliac anatomy ${ }^{1)}$. Therefore, pelvic angiograms should be considered in such cases. If active bleeding is suspected, urgent arteriography with embolization of the SGA must be considered prior to fasciotomy, even if there is increased risk of gluteal necrosis. Operative exploration and hemostasis in this region may be difficult due to the large overlying muscle group, hematoma, a possible delay in finding the injured vessel, and the possibility of causing more damage to the sciatic nerve and its branches. Moreover, blind attempts at clamping the vessel at its exit from the sciatic notch are not recommended as the sciatic nerve can easily be clamped along with these maneuvers ${ }^{2}$. Multiple authors have recommended performing angiography and embolization preopera- tively in a pelvic fracture model ${ }^{4}$.

\section{CONCLUSION}

We report a case of a patient of lumbar spine fracture associated with the SGA rupture absent pelvic fracture, and successfully treated with embolization before spinal surgery. Based on our case, we recommend that all patients with pelvic and lumbar vertebrae body fractures with direct injury on the buttock should routinely undergo CT scans with contrast media for early diagnosis of acute bleeding. We also wish to emphasize the importance of early internal iliac arteriography in the diagnosis and management of vascular injury associated with spinal burst fracture.

\section{ACKNOWLEDGMENT}

This work was supported by a grant of the Korea Health Technology Research and Development Project, Ministry for Health and Welfare Affairs (H114C3270).

This work was supported by a grant of the Korea Health Technology Research and Development Project, Ministry for Health and Welfare Affairs (HR16C0002).

\section{REFERENCES}

1. Agarwal M, Giannoudis PV, Syed AA, Hinsche AF, Matthews SJ, Smith RM: Pseudoaneurysm of the inferior gluteal artery following polytrauma: diverse presentation of a dangerous complication: a report of two cases. J Orthop Trauma 17:70-74, 2003 
2. Brumback RJ: Traumatic rupture of the superior gluteal artery, without fracture of the pelvis, causing compartment syndrome of the buttock. A case report. J Bone Joint Surg Am 72:134-137, 1990

3. Haikel S, Willett K: Traumatic rupture of the superior gluteal artery with a stable pelvic fracture. Injury 31:383-386, 2000

4. Hak DJ, Smith WR, Suzuki T: Management of hemorrhage in life-threatening pelvic fracture. J Am Acad Orthop Surg 17:447457, 2009

5. Kligman M, Mahrer A, Avi E, Roffman M: Hypotension as a delayed complication of rupture of a branch of the superior gluteal artery, following buttock contusion. Injury 33:285-287, 2002

6. Proschek R, Fowles JV, Bruneau L: A case of post-traumatic false aneurysm of the superior gluteal artery with compression of the sciatic nerve. Can J Surg 26:554-555, 1983

7. Sheridan MK, Blackmore CC, Linnau KF, Hoffer EK, Lomoschitz F, Jurkovich GJ: Can CT predict the source of arterial hemorrhage in patients with pelvic fractures? Emerg Radiol 9:188-194, 2002

8. Smith A, Chitre V, Deo H: Acute gluteal compartment syndrome: superior gluteal artery rupture following a low energy injury. BMJ Case Rep 2012, 2012

9. Smith K, Ben-Menachem Y, Duke JH, Jr., Hill GL: The superior gluteal: an artery at risk in blunt pelvic trauma. J Trauma 16:273279, 1976

10. Smyth NP, Rizzoli HV, Ordman CW, Khoury JN, Chiocca JC: Gluteal aneurysm. Arch Surg 91:1014-1020, 1965 